\title{
Organization of Excitatory Inputs from the Cerebral Cortex to the Cerebellar Dentate Nucleus
}

\author{
Yoshikazu Shinoda, Yuriko Sugiuchi and Takahiro Futami
}

\begin{abstract}
Intracellular recording was made from dentate nucleus neurons (DNNs) in anesthetized cats, to investigate cerebral inputs to DNNs and their responsible pathways. Stimulation of the medial portion of the contralateral pericruciate cortex most effectively produced EPSPs followed by long-lasting IPSPs in DNNs. Stimulation of the pontine nucleus (PN), the nucleus reticularis tegmenti pontis (NRTP) and the inferior olive (IO) produced monosynaptic EPSPs and polysynaptic IPSPs in DNNs. The results indicate that the excitatory input from the cerebral cortex to DNNs is at least partly relayed via the PN, the NRTP and the IO. Intraaxonal injection of HRP visualized the morphology of mossy fibers from the PN to the DN and the cerebellar cortex. The functional significance of the excitatory inputs from the PN and the NRTP to the DN is discussed in relation to the motor control mechanisms of the cerebellum.

RÉSUMÉ: Organisation des influx excitateurs originant du cortex cérébral vers le noyau dentelé. Des enregistrements intracellulaires ont été effectués dans des neurones du noyau dentelé (NNDs) chez les chats anesthésiés, pour investiguer les influx cérébraux vers les NNDs et les voies qui en sont responsables. La stimulation de la portion interne du cortex péricrucial contralatéral est la plus efficace pour produire des potentiels post-synaptiques excitateurs (PPSEs) suivis par des potentiels post-synaptiques inhibiteurs (PPSIs) dans les NNDs. La stimulation du noyau du pont (NP), du noyau du toit du quatrième ventricule (NTQV) et de l'olive inférieure (OI) produit des PPSEs monosynaptiques et des PPSIs polysynaptiques dans les NNDs. Ces résultats indiquent que les influx excitateurs provenant du cortex cérébral vers les NNDs sont transmis, au moins partiellement, par le NP, le NTQV et l'OI. L'injection intraaxonale de HRP a permis de visualiser la morphologie des fibres moussues du NP vers le ND et le cortex cérébelleux. Nous discutons de la signification fonctionnelle des influx excitateurs du NP et du NTQV vers le ND, en relation avec les mécanismes du contrôle moteur du cervelet.
\end{abstract}

Can. J. Neurol. Sci. 1993; 20 (Suppl. 3): S19-S28

The cerebellar cortex receives two types of afferent fibers from outside the cerebellum; the mossy fibers and the climbing fibers. ' Both of these afferent fibers mediate excitatory inputs to the cerebellum. ${ }^{2}$ Deep cerebellar nucleus neurons and vestibular nucleus neurons give rise to output fibers that convey excitatory signals to other targets. These nuclei are impinged upon by inhibitory inputs from Purkinje cells in the cortex. ${ }^{3,4}$ It has been generally assumed that the excitatory inputs to the deep cerebellar nuclei are supplied through collaterals of afferents to the cerebellar cortex. In spite of a wealth of anatomical papers reporting the afferent pathways to the cerebellar cortex, there were far fewer studies on afferent projection to the deep cerebellar nuclei until recently. ${ }^{2.5-8}$ As for the projections from the precerebellar nuclei to the dentate nucleus (DN), ${ }^{9,10}$ the existence of the projection from the pontine nucleus (PN) to the DN is most controversial. Dietrichs et al." could not positively demonstrate the afferent projection from the PN to the DN with a wheat germ agglutinin-horseradish peroxidase (HRP) injection method. Therefore, they doubted the results obtained using HRP as a retrograde tracer, since massive fibers projecting to the cerebellar cortex in and outside the DN damaged by an injection needle might take up HRP leaking along an electrode track. Cerebral inputs to the DN and the interpositus nucleus (IN) have been examined by Allen et al., ${ }^{12.13}$ using an extracellular recording method. Although both facilitation and depression of spike activities were induced in dentate nucleus neurons (DNNs) by stimulation of various parts of the cerebral cortex, it was not possible to determine whether facilitation of spike activities was caused by either excitatory postsynaptic potentials (EPSPs) or disinhibition, and their depression by either inhibitory postsynaptic potentials (IPSPs) or disfacilitation. The only previous intracellular recording from DNNs was reported by lto et al. ${ }^{4}$ They found that stimulation of the ipsilateral PN produced monosynaptic EPSPs with a very small amplitude in 5 DNNs in the cat. Dietrichs et al." were doubtful of that electrophysiological result, since it was not clear from that report whether

From the Department of Physiology, School of Medicine, Tokyo Medical and Dental University, Tokyo, Japan

Reprint reuqests to: Dr. Yoshikazu Shinoda, Department of Physiology, School of Medicine, Tokyo Medical and Dental University, 1-5-45, Yushima, Bunkyo-ku, Tokyo, Japan 
stimulus effects were localized in the PN. The cerebellar projection from the contralateral PN and nucleus reticularis tegmenti pontis (NRTP) is dominant and the fibers from these nuclei pass through the ipsilateral PN. Therefore, the origin of the monosynaptic EPSPs evoked by stimulation of the ipsilateral PN remains to be determined.

The present study was undertaken to analyze excitatory inputs to DNNs from the cerebral cortex and to determine the pathways from the cerebral cortex to the $\mathrm{DN}$, especially to determine the presence or absence of the excitatory projection from the PN, the NRTP and the inferior olive (IO) to the DN. The results show that monosynaptic connections indeed exist from the contralateral PN, NRTP and IO to the DN, and that the excitatory inputs from the cerebral cortex are conveyed to the DN via these precerebellar nuclei. A part of this study was reported before. ${ }^{14}$

\section{METHODS}

Experiments were performed on cats weighing $2.8-4.3 \mathrm{~kg}$. The animals were anesthetized with sodium pentobarbital (Nembutal, Abott, Switzerland; $35 \mathrm{mg} / \mathrm{kg}$ and supplemented as required), and were mounted in a stereotaxic apparatus. The animals were paralyzed by intravenous injection of pancuronium bromide (Mioblock, Organon Co.) and artificially ventilated. A craniotomy was performed to expose the cerebral cortex, and stimulating electrodes (concentric bipolar metal electrodes) were inserted into the red nucleus (RN), and the cerebral peduncle (CP) or areas 4 and 6 on the left side (Figure 1 inset). The occipital bone was removed and stimulating electrodes of the same type were stereotaxically placed in the PN and the IO on the left side.

Cerebral influences on DNNs were investigated with intracellular recording from DNNs by stimulating the cerebral cortex and the CP. An array of 9 concentric electrodes was positioned in the gray matter of the precruciate gyrus and the lateral portion of the postcruciate gyrus (Figure 2A). For PN stimulation, 2 or 3 concentric electrodes were inserted stereotaxically from the dorsal surface of the cerebellar cortex into the target $(A=2.0 \mathrm{~mm}$ $\mathrm{P}=2.0 \mathrm{~mm} ; \mathrm{L}=0.3-1.5 \mathrm{~mm})$ at an angle of about $30^{\circ}$ inclined posteriorly in the sagittal plane. For systematic mapping of the effective stimulating sites in the PN and NRTP, a contact array of five fine wires was made by gluing together $200 \mu \mathrm{m}$ wires insulated except for the exposed tips ( $0.3 \mathrm{~mm}$ exposure), with their exposed ends separated from one another by $0.6-1.3 \mathrm{~mm}$ longitudinally. Two arrays of these multipolar electrodes were positioned in the PN and NRTP (see Figure 3C). The stimulating electrode positions in the PN were verified histologically. ${ }^{15}$ To examine the influence of the IO on DNNs, two concentric

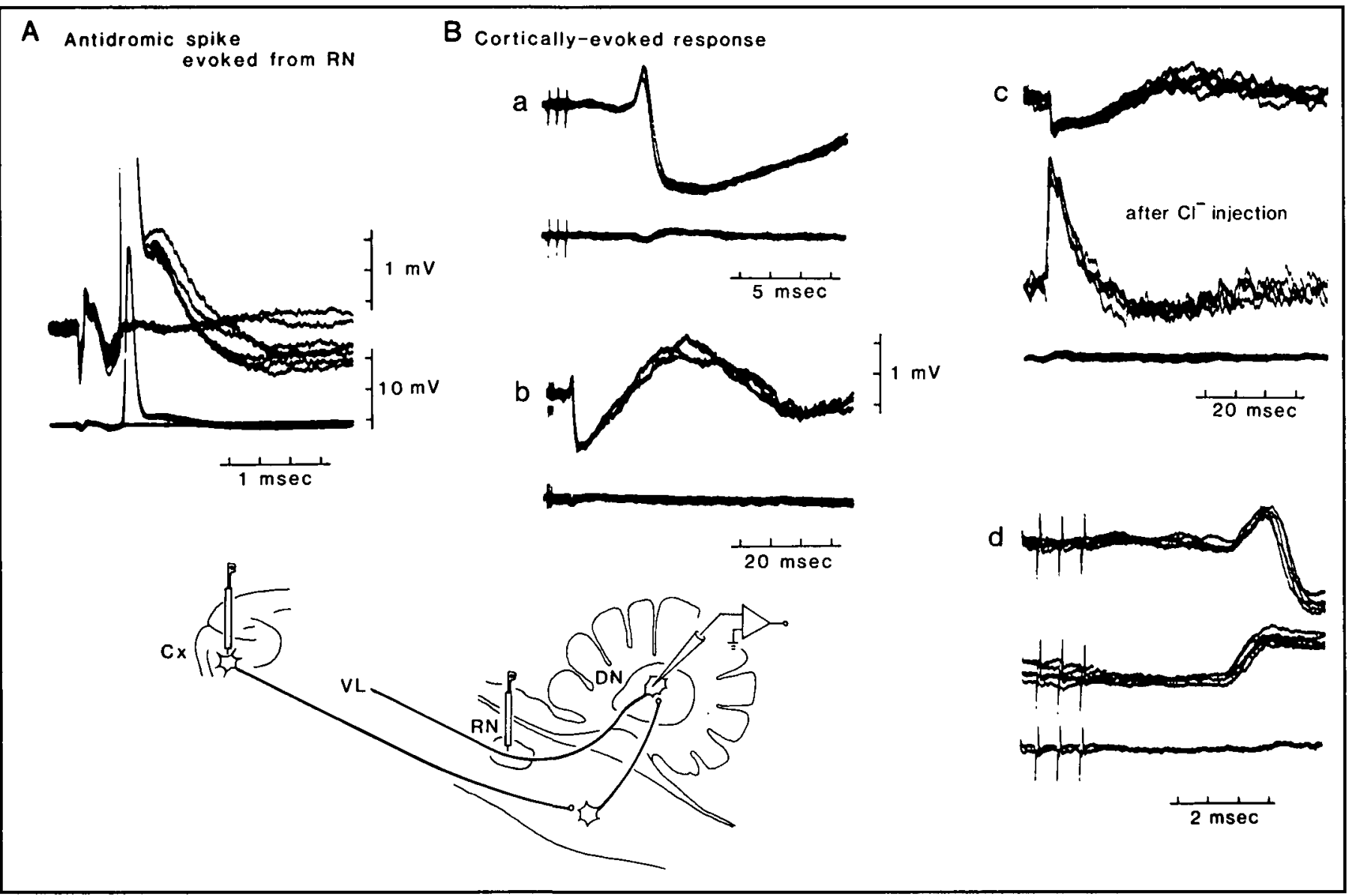

Figure 1-Cerebral inputs to single DNNs. A: Antidromic spikes evoked in an all-or-none manner in response to threshold straddling stimulation $(200 \mu \mathrm{A})$ in the RN. B: Intracellular records from a DNN following stimulation of the cerebral cortex. a-b: Postsynaptic potentials of depolarization-hyperpolarization sequence evoked from the contralateral area 6 at fast (a) and slow sweep speeds (b) (stimulating site 3 in Figure $2 A$ ). C: Reversed IPSPs recorded after Cl injection into the cell by hyperpolarizing current. D: Early EPSPs remained unchanged after Cl injection. 
electrodes were positioned in the center or the medial border of the IO.

For stimulation, rectangular negative pulses of $0.2 \mathrm{msec}$ duration were applied bipolarly through individual concentric electrodes placed in the cerebral cortex, the CP, the RN, the PN and the IO or monopolarly through each electrode of the electrode arrays in the PN and the NRTP relative to an indifferent electrode in the temporal muscles. For cortical stimulation, single pulses of $0.5-1 \mathrm{~mA}$ were not always effective to evoke responses in DNNs, and so double or triple pulses at $1.5 \mathrm{msec}$ intervals were usually used. For stimulation of the PN and the NRTP, the currents of $500 \mu \mathrm{A}$ or less were applied with concentric electrodes, and the currents of $300 \mu \mathrm{A}$ or less with monopolar electrodes. Intracellular recording was made from DNNs on the right side with glass micropipettes filled with $3 \mathrm{M} \mathrm{KCl}(15$ $25 \mathrm{M} \Omega$ in resistance).

At the end of each experiment, electrolytic lesions were made by passing negative currents of $30 \mu \mathrm{A}$ for $20 \mathrm{sec}$ through each stimulating electrode. The animal was perfused with $10 \%$ formaldehyde and the brain was removed for later histological examination. The locations of lesions were determined on 100 $\mu \mathrm{m}$ serial frozen sections of the brain stem stained with cresyl violet. In HRP staining experiments, the animal was perfused with $0.1 \mathrm{M}$ phosphate buffer solution and then with a mixture of $1 \%$ glutaraldehyde and $2 \%$ paraformaldehyde in $0.1 \mathrm{M}$ phosphate buffer ( $\mathrm{pH} 7.4$ ). ${ }^{16}$ Serial coronal frozen sections of 100 $\mu \mathrm{m}$ thicknesss were treated for HRP, using the diaminobenzidine method. Three dimensional reconstruction of the neuronal trajectory was performed on these serial sections.

\section{RESULTS}

To analyse cerebral inputs to the DN and their pathways, intracellular recording was made from 201 efferent DNNs, in which postsynaptic potentials (PSPs) were examined by stimulation of various portions of the cerebral cortex and the

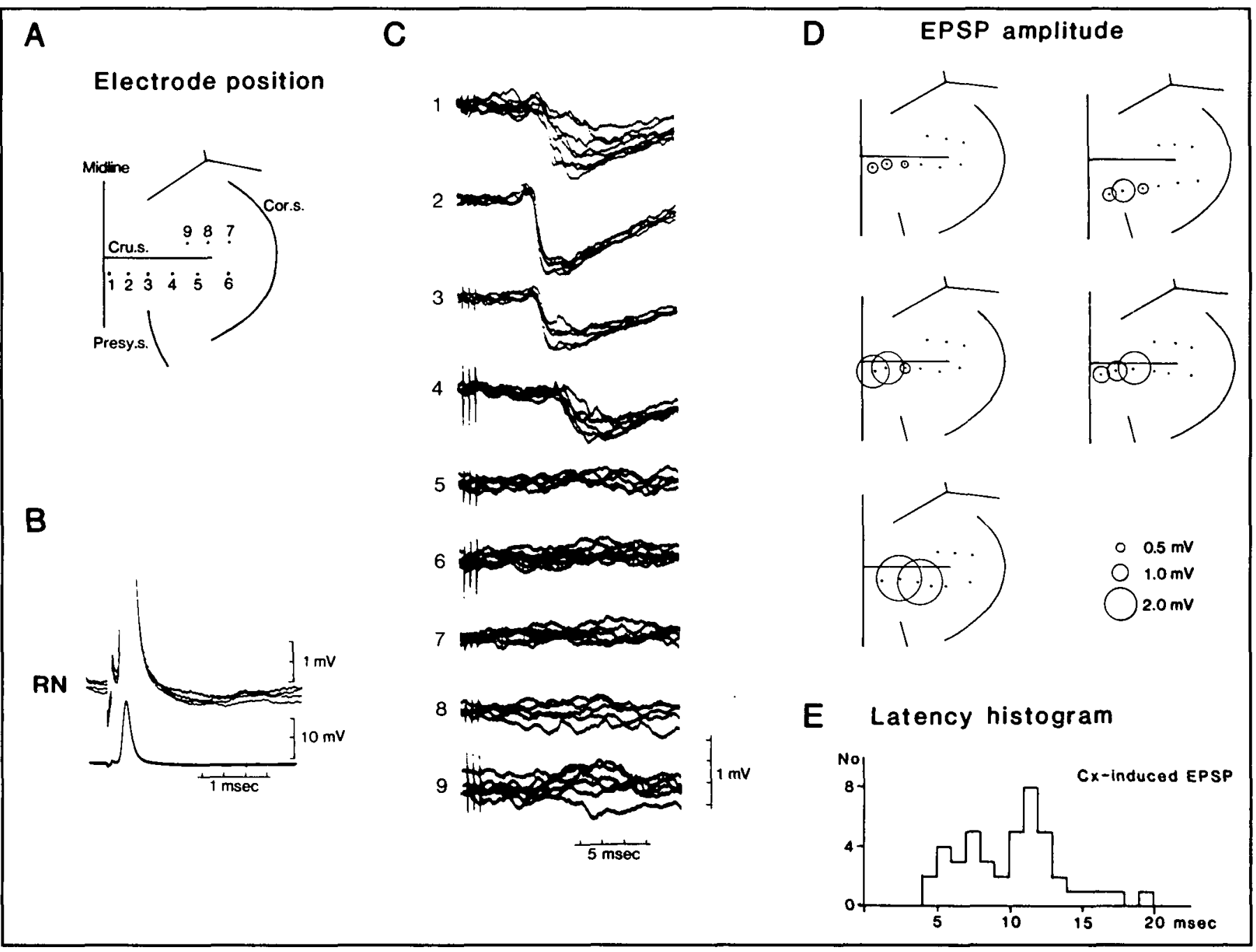

Figure 2 -Distribution of effective stimulating sites in the cerebral cortex to induce PSPs in DNNs. A: Arrangement of 9 stimulating electrodes in the pericruciate cortex. cru.s.; cruciate sulcus. presy.s.; presylvian sulcus. cor.s.; coronal sulcus. B: Antidromic spikes evoked by stimulation of the RN at suprathreshold. C: Distribution of cortical inputs to a single DNN. Responses to stimulation of the cortical sites (1-9) indicated in A. PSPs were only evoked from the most medial four electrodes. D: Diagrams showing the distribution of effective sites for evoking EPSPs in 5 DNNs. The diameter of a circle is proportional to the amplitude of an EPSP evoked from each cortical site. Dots without circles represent the sites from which we failed to evoke EPSPS. A train of three pulses at I mA was used to evoke EPSPS. E: Latency histograms of cerebral-evoked EPSPs in DNNs. 
precerebellar nuclei. Efferent DNNs were identified by their antidromic responses to stimulation of their output fibers within the contralateral RN (Figure lA). ${ }^{17.18}$ Out of 143 DNNs examined on their caudal projection, $69 \mathrm{DNN}$ s were activated antidromically only from the RN, 54 (37.8\%) DNNs were activated antidromically from the PN or the NRTP, but not from the IO and $20(14.0 \%)$ DNNs were antidromically activated from the IO.

\section{Cerebral Inputs to Dentate Nucleus Neurons}

Cerebral effects on DNNs were investigated by stimulating areas 6 and 4 on the contralateral side. Stimulation of the medial portion of the precruciate gyrus produced depolarization followed by long-lasting hyperpolarization (Figure $1 \mathrm{Ba}$ ). This hyperpolarization was further followed by long-lasting depolarization (Figure $\mathrm{lBb}$ ). Iontophoretic injection of $\mathrm{Cl}^{-}$into a DNN reversed the hyperpolarizing potential to the depolarizing poten- tial and its following depolarizing potential to the hyperpolarizing potential (Figure $\mathrm{IBc}$ ), but the early depolarization remained unaffected (Figure 1Bd). Therefore, we concluded that the early depolarization was an EPSP, the long-lasting hyperpolarization was an IPSP and the late depolarization was disinhibition. ${ }^{19}$

To determine the effective cortical area able to induce responses in DNNs, the stimulating electrodes were arranged in such a way as shown in Figure $2 \mathrm{~A}$ and the effective stimulating sites were determined from which EPSPs could be evoked at a stimulus intensity of $1 \mathrm{~mA}$. In a typical example shown in Figure 2C, shortest-latency and largest-amplitude EPSPs and IPSPs were evoked from the position 2, and slightly smallerand longer-latency EPSPs and IPSPs were evoked from the adjacent positions 1 and 3. Much longer-latency IPSPs were evoked from the position 4 . In five other examples shown in Figure 2D, the effective stimulating sites were mainly found in the medial portion of the precruciate gyrus, which corresponds

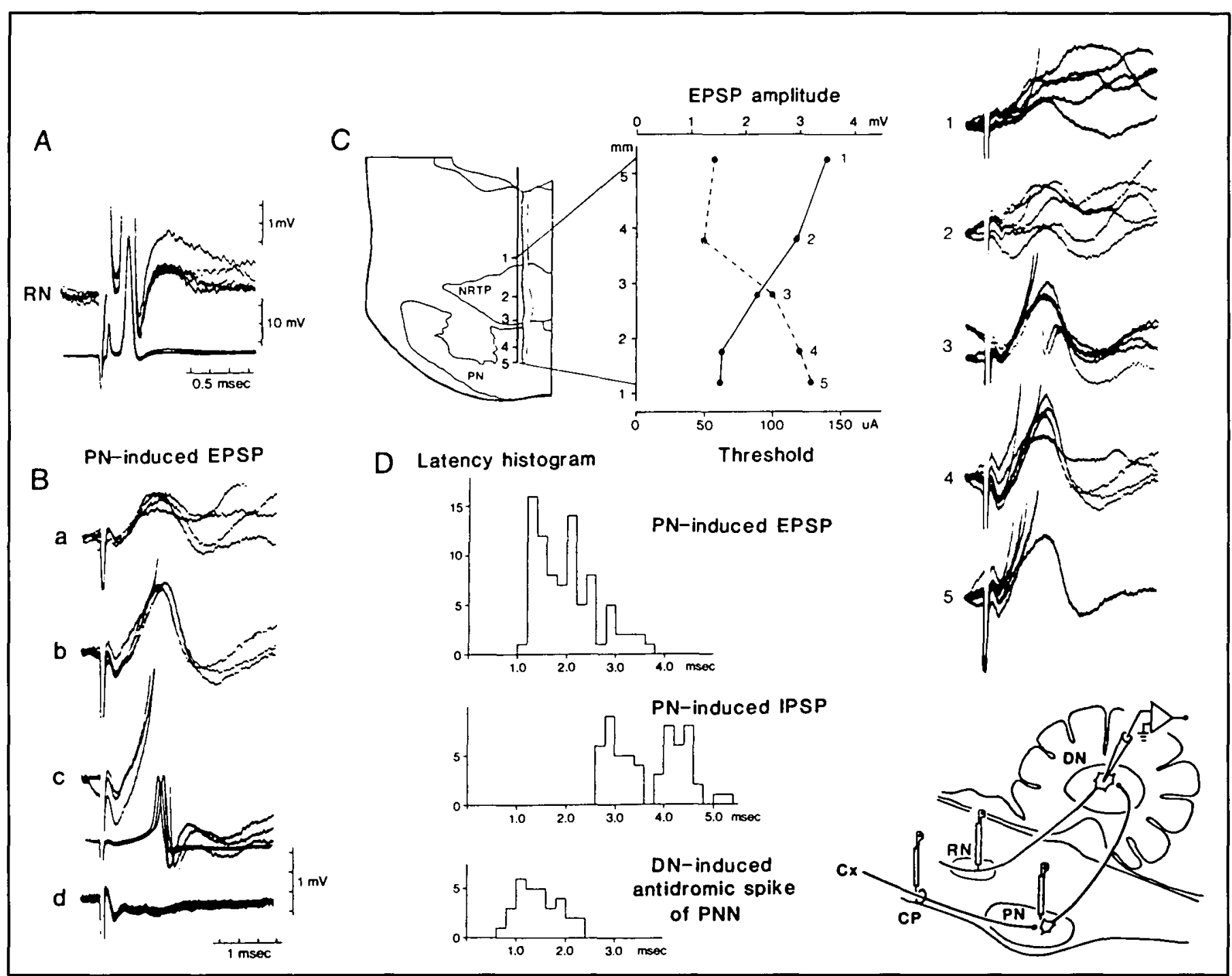

Figure 3-Inputs from the PN to DNNs. A: Antidromic spikes in response to stimulation in the RN. B: Effects of stimulus strength on the responses of a DNN. Stimulus intensity increased from $100 \mu \mathrm{A}(\mathrm{a})$ to $200 \mu \mathrm{A}$ (b) and $300 \mu \mathrm{A}(\mathrm{c})$. C: Transverse section through the pons angled at $38^{\circ}$ posteriorly to the vertical plane, showing the relationship between the amplitude (dotted line) and the threshold (solid line) of the EPSPs relative to stimulating sites in the PN and NRTP. D: Latency histograms of EPSPs and IPSPs evoked in DNNs from the PN and extracellular spikes of PNNs evoked from the $D N$. 
to area $6 a \beta$ and its adjacent area $4 \gamma$ of Hassler and MuhsClement, ${ }^{20}$ although weak responses were evoked from the lateral portion of the precruciate gyrus in some DNNs. The shortest latency of the cortically-evoked EPSPs for individual DNNs ranged from 4.0 to $20.4 \mathrm{msec}$ (mean \pm S.D., $9.7 \pm 3.5 \mathrm{msec}, \mathrm{N}=$ 44) (Figure 2E) and that of the cortically-evoked IPSPs ranged from 8.0 to $27.0 \mathrm{msec}(13.4 \pm 2.6 \mathrm{msec}, \mathrm{N}=49)$.

\section{Inputs From the PN and the NRTP to Dentate Nucleus Neurons}

To determine the pathways from the cortex to the DN, we searched for the precerebellar nuclei to mediate the cortical inputs to the DN. Effects of PN stimulation on DNNs will be described first. Stimulation of the contralateral PN evoked short-latency EPSPs followed by IPSPs (Figure 3B). The EPSPs had latencies of $1.1-3.6 \mathrm{msec}$ (mean \pm S.D., $2.0 \pm 0.6 \mathrm{msec}, \mathrm{N}$ $=84)$ and the IPSPs had latencies of $2.6-5.3 \mathrm{msec}(3.7 \pm 0.7$ msec, $N=58$ ) (Figure $3 D$ ). To identify the $P N$ as a site responsible for generation of the EPSPs in DNNs, systematic mapping for effective stimulating sites in the PN and the NRTP was performed. Figure $3 \mathrm{C}$ shows the stimulated sites in the $\mathrm{PN}$ and NRTP. When a DNN was penetrated, the most effective site in the PN or the NRTP was sought by measuring the amplitudes of EPSPs evoked at the same stimulus intensity, and the thresholds for the EPSPs in individual stimulating sites. In the DNN exemplified in Figure 3C, stimulation of the NRTP produced smallamplitude EPSPs in the DNN and the thresholds for the EPSPs were high. When the more ventral portion of the PN was stimulated, larger EPSPs were evoked and the thresholds for the EPSPs became much lower. In this way, the lowest threshold site and the maximum response site to evoke EPSPs were identified within the PN for 39 DNNs.

To demonstrate that cerebral signals are conveyed to DNNs via PN neurons (PNNs), we used a conditioning-testing paradigm and examined the interaction between $\mathrm{CP}$-evoked and PN-evoked EPSPs. The existence of spatial facilitation was examined in the DNNs with monosynaptic EPSPs evoked from the PN. Stimulus intensities to the CP and the PN were decreased and the time of the PN stimulus was delayed so that the EPSPs from the CP and the PN were evoked in the DNN at the same onset. Combined stimulation of the CP and the PN evoked much larger EPSPs than the sum of the control EPSPS evoked separately by the CP and the PN (not illustrated). This spatial facilitation should occur only when the PN stimulus activates cell bodies of PNNs rather than their axons. Similar spatial facilitation was observed in 5 out of 8 DNNs. Therefore, we conclude that there is an excitatory input to DNNs from the cerebral cortex by way of the PN.

To examine whether neurons in the NRTP terminated on DNNs or not, effective stimulating sites were carefully sought in the NRTP in the same experimental arrangement as in the PN. In an example shown in Figure 4F, the thresholds of EPSPs were plotted at individual stimulating sites for 13 DNNs. The most effective stimulating site for the monosynaptic EPSP was found in the NRTP and/or the PN in individual DNNs. Twentyone DNNs were identified as receiving EPSPs from the NRTP, since they were evoked from the electrode in the NRTP with the lowest threshold. The latencies of the EPSPs were 1.1-2.5 msec $(1.6 \pm 0.4 \mathrm{msec}, \mathrm{N}=21)$ and those of the IPSPs were 2.7 $4.5 \mathrm{msec}$ ( $3.4 \pm 0.7 \mathrm{msec}, \mathrm{N}=11$ ). The spatial facilitation between CP-evoked EPSPs and NRTP-evoked monosynaptic EPSPs was observed in 3 out of 5 DNNs.

\section{Inputs From the IO to Dentate Nucleus Neurons}

Stimulation of the contralateral IO produced long-latency depolarization followed by long-lasting hyperpolarization in a DNN (Figure 4Aa, Ba). Injection of $\mathrm{Cl}^{-}$into the cell reversed the late long-lasting hyperpolarization to the depolarizing potential, but the early depolarization remained unchanged (Figure $4 \mathrm{Ab}$ and $\mathrm{Bb}$ ). Therefore, these potentials were considered to be EPSPs and IPSPs, respectively. Around the peak of the IPSPs, notches were occasionally found. This indicates that there are at least two synchronous inhibitory presynaptic bombardments to DNNs. With the increase of stimulus intensity, the amplitude of EPSPs gradually increased and orthodromic spikes were generated, when the EPSPs exceeded firing threshold (Figure 4C). The latencies of the EPSPS, however, remained constant even near the threshold, strongly suggesting that these EPSPs were induced monosynaptically from the contralateral IO. The latency of the EPSPs evoked from the IO ranged from 2.0 to $5.9 \mathrm{msec}$ (mean \pm S.D., $3.4 \pm 0.8 \mathrm{msec}, \mathrm{N}=121$ ). In order to determine that some of the EPSPs evoked from the cerebral cortex were induced by way of the IO, interaction of IO-evoked EPSPs and CP-evoked EPSPs was examined in a DNN. Conditioning stimulation of the CP facilitated the monosynaptic EPSPs evoked from the IO (not illustrated). The existence of this spatial facilitation of the inputs strongly suggested that the CP-evoked EPSPs were partly mediated by way of the 10. The latency of the IPSPs evoked from the contralateral IO ranged from 3.6 to $8.4 \mathrm{msec}(5.5 \pm 0.9 \mathrm{msec}, \mathrm{N}=133)$. Many IPSPs had latencies between 5.0 and $7.0 \mathrm{msec}$. These IPSPs were assumed to be produced by way of Purkinje cells, since climbing fibers originating from the IO excite Purkinje cells in the cerebellar cortex ${ }^{2}$ and Purkinje cells, in turn, project to cerebellar nucleus neurons. ${ }^{4}$

Single DNNs were often found to receive convergent inputs from the PN, the NRTP and the IO as shown in Figure 4D - E. The input from the IO was most prominent and most stable among three inputs and the latencies of the EPSPs evoked from the NRTP (Figure 4D) or the PN (Figure 4E) were much shorter than those from the IO (Figure $4 \mathrm{C}$ ) in individual DNNs.

\section{The Morphology of Single PN Axons in the DN}

To confirm the existence of mossy fiber projections from the PN to the DN morphologically, mossy fibers were visualized by intraaxonal staining with HRP. ${ }^{16}$ Single axons were penetrated in the white matter of the cerebellum and were identified electrophysiologically as PNN axons by their direct responses to stimulation of the contralateral PN, their synaptic activation from the contralateral cerebral cortex and the decrease in threshold for evoking direct spikes in stimulation of the PN by conditioning stimuli applied in the cerebral cortex. After this electrophysiological identification, HRP was iontophoretically injected into the axons. Figure 5 shows an example of the axonal trajectory of axon collaterals of a mossy fiber arising from the contralateral PN. The stem axon of this PNN was traced to the granular layer of the cerebellar cortex and it terminated there as typical mossy fiber rosettes. On its way, the axon collaterals were given off to the DN. The diameters of these collaterals were very thin compared with the large diameter of the parent 
axon. These results provided morphological evidence for the existence of the projection from the PN to the DN.

\section{Discussion}

\section{Excitatory Inputs From the Precerebellar Nuclei to the DN}

The present study demonstrated that at least three precerebellar nuclei, the PN, the NRTP and the IO, convey excitatory inputs to DNNs. Localized stimulation of the PN and the NRTP evoked short-latency EPSPs in DNNs (Figures 3 and 4). These EPSPs were monosynaptic for the following reasons; 1) short latencies ranging from 1.1 to $2.7 \mathrm{msec}$ (Figures 3D and 4D, E), 2) constant latencies even at near-thresholds (Figure 3B) and 3) latencies that were compatible with antidromic latencies of PNNs evoked from the contralateral DN $(0.7-2.4 \mathrm{msec})$, making an allowance of $0.3-0.4 \mathrm{msec}$ for a synaptic delay (Figure 3D). The most effective stimulus sites in the PN and NRTP were determined by measuring thresholds for evoking EPSPs and amplitudes of the EPSPs and the threshold minima were found in the PN or the NRTP or both and the lowest thresholds were as low as $20 \mu \mathrm{A}$. Taken together, the results indicated that neurons in the PN and the NRTP project to the DN. The morphological study confirmed the projection from the PN to the DN (Figure $5),{ }^{16}$ and further showed that this projection is by way of collaterals of mossy fibers terminating in the cerebellar cortex.

A third source of the excitatory input to the DN is the IO. Electrophysiologically, Ito et al. ${ }^{4}$ found that stimulation of the contralateral IO produced EPSPs in DNNs. The present study confirmed their result and also the result obtained by Amatuni et al. ${ }^{21}$ with an extracellular recording method. These EPSPs are most likely to be mono- synaptic for the following reasons. 1) The latencies of the EPSPs were constant, when the stimulus strength was varied (Figure 4C). 2) Antidromic spikes of olivary neurons were evoked from the cerebellar cortex at a latency from 2.8 to $5.5 \mathrm{msec}^{22}$ Judging from the small diameter of climbing fibers and the slow conduction velocity of the antidromic spikes, the latency of the IO-induced EPSPs (2.0 $5.9 \mathrm{msec}$ ) is compatible with the latency of the monosynaptic

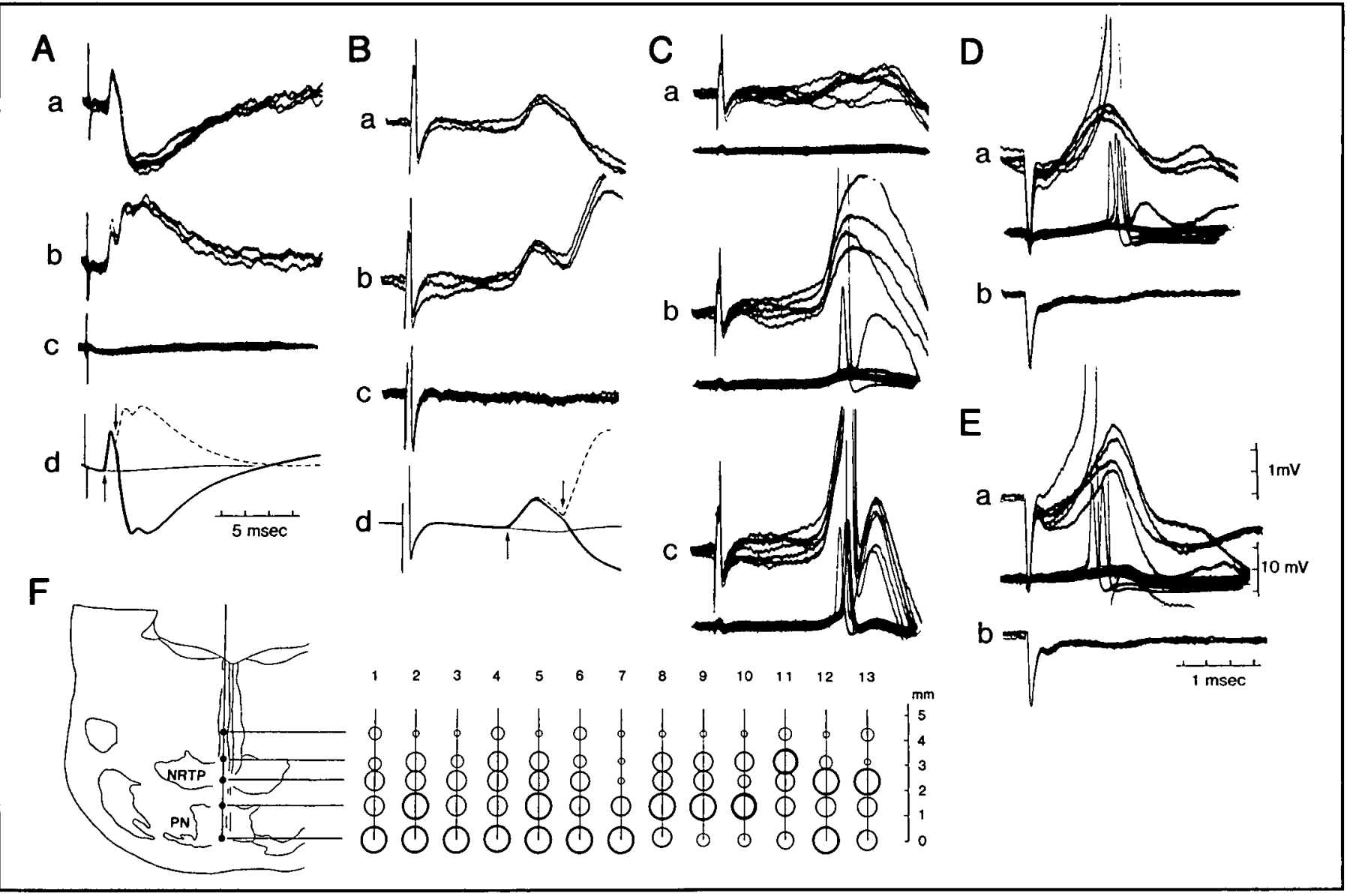

Figure 4-Intracellular responses of DNNs to stimulation of the IO. A: Responses to IO stimulation at a long time scale. a: Control. b: Reversal of hyperpolarization after iontophoretic injection of $\mathrm{Cl}$. c: Field potential recorded just outside the cell. d: Superposition of three traces (a, b, c) to determine the onsets of EPSPs and IPSPS. The upward and the downward arrow's indicate the onsets of the EPSPS and the IPSPs, respectively. B: Long-latency IPSPs evoked from the IO. a: Intracellularly-recorded IO-evoked EPSPs and IPSPs. b: The reversed IPSPs after Cl injection. C: Field potentials. d: Determination of the onsets of the EPSPS (upward arrow) and the IPSPS (downward arrow) by superimposing the three traces shown above. C: Responses evoked by stimulation of the contralateral $1 O$ and effects of the stimulus strength on PSPs. Stimulus intensities; 600 $\mu A(a), 650 \mu A(b)$, and $900 \mu A(c)$. Time calibration in $E$ is applied to all traces except for $A$. D-E: Stimulation of the NRTP $(D)$ and the PN (E) evoked EPSPs followed by IPSPs with superimposed orthodromic spikes in some traces $(a)$. $b$ : Juxtacellular field potentials. F : Effects of stimulation of the PN and the NRTP in 13 DNNs. Five sites illustrated in the pons on the left were stimulated. The largest and the smallest circles indicate the thresholds for the EPSPs at $50 \mu \mathrm{A}$ and $300 \mu \mathrm{A}$, respectively. 


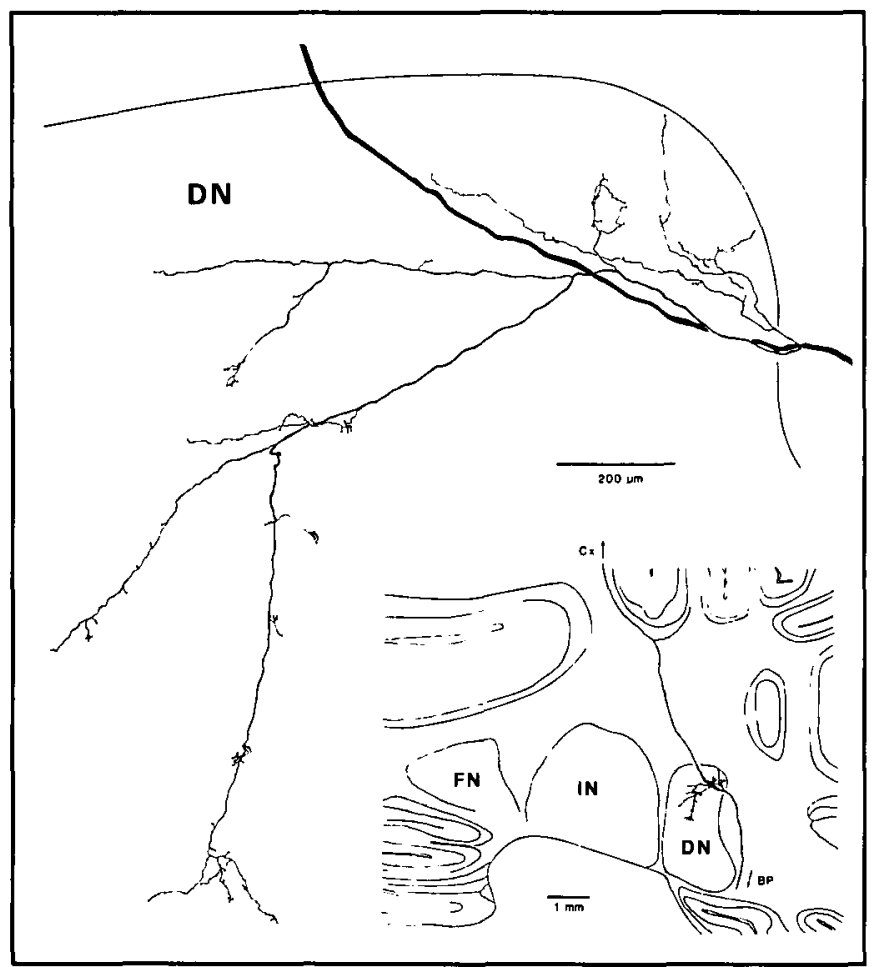

Figure 5-Reconstruction of two axon collaterals arising from a single node of Ranvier of a parent $P N$ axon stained intraaxonally with HRP. The inset on the right shows the axonal trajectory of the parent mossy fiber axon.

EPSPs. In fact, the projection from the IO to the DN has been anatomically suggested ${ }^{8}$ and recently confirmed with the autoradiographic method. ${ }^{23}$ This projection is completely contralateral, ${ }^{24}$ and there seems to be a topographical arrangement between the IO and the cerebellar nuclei; the DN receives the input from the principal olive and the posterior IN and the anterior IN receive inputs from the dorsal and the medial accessory olives, respectively. ${ }^{5.7}$

\section{Cerebral Inputs to the DN}

The present study showed that DNNs receive inputs mainly from the medial portion of the precruciate gyrus (area $6 \mathrm{a} \beta$ ) and its adjacent middle portion of the precruciate gyrus (area $4 \gamma$ ). This result is partly in agreement with the results obtained in the monkey, ${ }^{13}$ in that the DN and the IN receive main inputs from the premotor and the supplementary motor regions of area 6 , and the sensorimotor cortex (area 4 and the first somatosensory cortex), respectively. Allen et al. ${ }^{13}$ also found that there was a great deal of convergence of the inputs from areas 4 and 6 to DNNs. However, convergence of the inputs from areas 4 and 6 to single DNNs was rare in the present study. This discrepancy between our results and Allen et al.'s result might be due to several factors (see the details in Shinoda et al. ${ }^{14}$ ).

The neural pathways mediating EPSPs and IPSPs to the DN are assumed to be multifold. The $\mathrm{PN}$ receives cerebral inputs from all cortical areas and there is a topographical relationship. ${ }^{25-27}$ The NRTP also receives inputs from all cortical areas. ${ }^{28,29}$ Cortical inputs to PNNs were extensively analysed electrophysiologically. ${ }^{30-33}$ The majority of PNNs are innervated by axon collaterals of PTNs. ${ }^{32}$ In previous studies, differential inputs from areas 4 and 6 to single PNNs have not been analysed. In a recent study of corticofugal neurons in areas 4 and 6 , it became clear that there are two groups of PN-projection neurons; corticopontine neurons (CPNs) and PTNs with axon collaterals to the PN. ${ }^{34} \mathrm{CPNs}$ in areas 6 and 4 make up $64.6 \%$ and $30.4 \%$ of the total PN-projecting neurons, respectively. The latency of EPSPs evoked in DNNs from area 6 was fairly long $(4.0-20.5 \mathrm{msec}$ ) (Figure 2F). This long latency of the EPSPs is partly explained by the finding that, among PN-projecting neurons, slow CPNs are predominant in area $6 .{ }^{34}$ Furthermore, slow PNNs receive inputs from slow PN-projecting corticofugal neurons and fast PNNs receive inputs from fast PN-projecting neurons. ${ }^{31,33}$ Consequently, PNNs receiving the input from area 6 must have slow conducting axons. Therefore, PNNs with slow conducting axons may be another cause for the longer latency of the EPSPs produced in DNNs following cortical stimulation. The IO also receives cortical inputs. ${ }^{35-37}$ Recent studies suggested the relatively greater contribution of area 6 to olivary afferents compared with area $4 .{ }^{38.39}$ Stimulation of the precruciate cortex induces spikes in inferior olivary neurons at a latency of $8-20$ msec. ${ }^{22.40}$ This response may be mediated by two pathways; a direct cortico-olivary and an indirect cortico-rubro-olivary pathway. ${ }^{40}$ Since olivary neurons are activated at a long latency from the cortex, the EPSPs and the IPSPs evoked from the cerebral cortex with the shorter latencies could not be mediated via the IO, but those with longer latencies might be mediated via the IO.

\section{Functional Consideration of Excitatory Inputs to the DN}

During the slow tracking movement of the wrist flexion and extension, both Purkinje cells ${ }^{41}$ and neurons in the DN and $\mathrm{IN}^{42}$ were found to show a bidirectional increase of spike activities rather than a reciprocal pattern. In rapid alternating movements of a forelimb, nuclear cells showed reciprocal changes of activities above and below a resting mean frequency, 43,44 whereas Purkinje cells had a bidirectional discharge pattern (predominantly bidirectional increase)..$^{41}$ The neural mechanism of this increase of discharge frequency in cerebellar nuclear cells is an unsolved important question of cerebellar physiology. There are two possible pathways to produce this facilitation (Figure 6). If there is no excitatory input to the DN, nuclear cell firing rates will be caused to change solely by changes in Purkinje cell firing rates; an increase in nuclear cell discharge will be caused by disinhibition through Purkinje cells (Figure 6A). On the other hand, if there is an excitatory input of extracerebellar origin to the DN, it will cause an initial increase of nuclear cell discharge (Figure 6B). Since Purkinje cells are inhibitory, ${ }^{3}$ a decrease of their activity will produce an increase in nuclear cell discharge by disinhibition. However, most Purkinje cells increase their activity at the onset of movement. ${ }^{41.43}$ Accordingly, other excitatory inputs of extracerebellar origin must exist to increase nuclear cell discharge at the onset of movement. In many textbooks, the projections of axon collaterals of mossy fibers or climbing fibers to the deep cerebellar nucleus had been described in spite of no experimental data until recently. Allen and Tsukahara ${ }^{45}$ were very thoughtful, because they did not include the projection of mossy fibers to the deep cerebellar nucleus in their diagrams after very extensive review of the literature. The existence of the projections of mossy fibers to the cerebellar nucleus had been controversial. ${ }^{11.46-48}$ However, we demonstrated that 


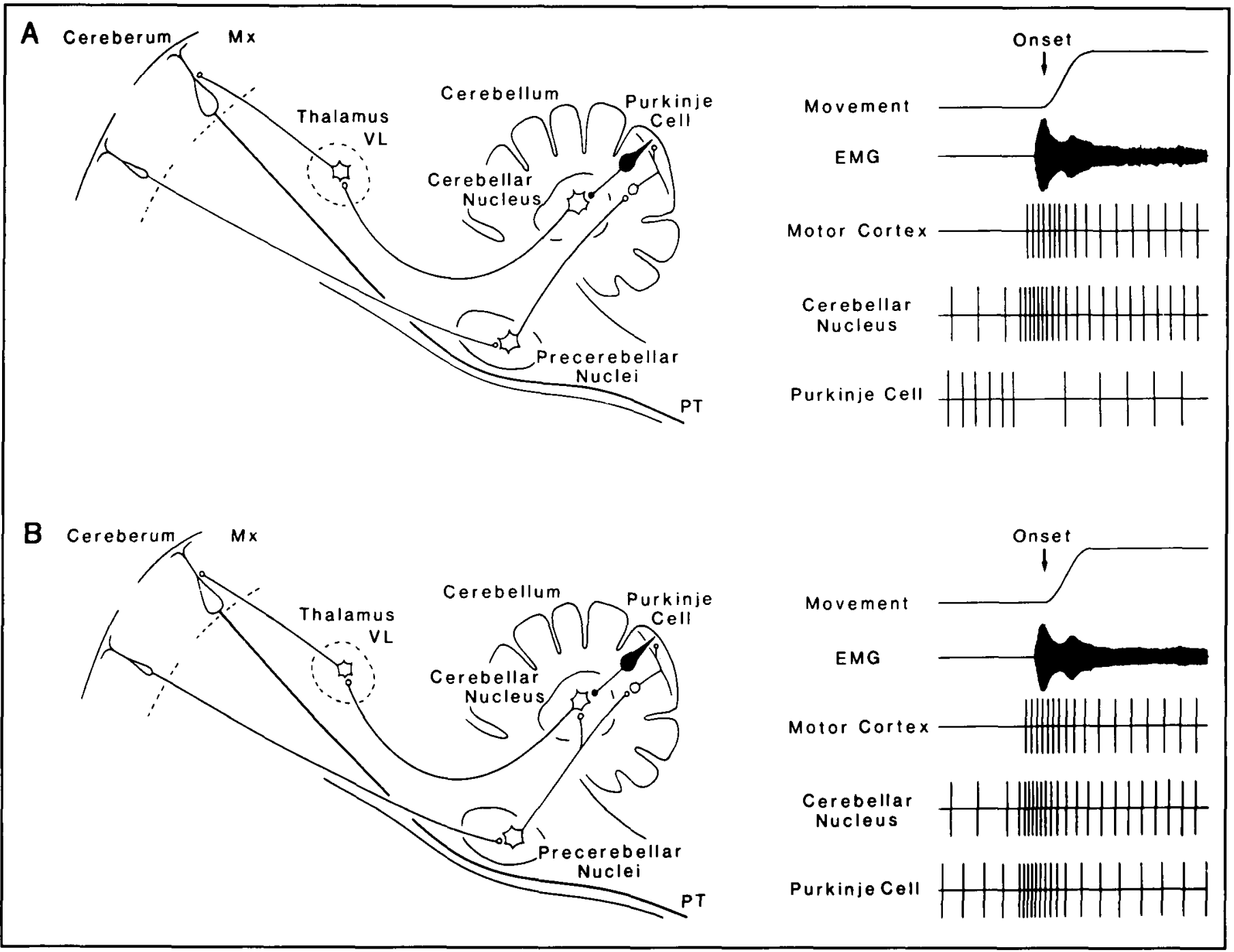

Figure 6 - Two possible operational modes of cerebellar corticonuclear interaction in the DN in relation to mossy fiber inputs from the cerebral cortex.

there are excitatory pathways from the PN, the NRTP, and the IO to the DN. ${ }^{14}$ Our study has also given morphological evidence for the existence of mossy fiber collaterals from PN axons to the DN. ${ }^{16}$ Gerrits and Voogd ${ }^{49}$ clearly demonstrated the projection from the NRTP to the DN. Therefore, these results suggest that excitatory inputs from the PN and the NRTP to the DN could at least partly contribute to the increase of activity of cerebellar nucleus neurons. In addition to these excitatory inputs, signals conveyed from these precerebellar nuclei exert their modulatory influences onto efferent DNNs via Purkinje cells. These Purkinje cells could impose efficient modulation of activity on nuclear cells by inhibition or disinhibition.

The question remains as to whether the input by way of these mossy fiber collaterals or climbing fibers is strong enough to change neuronal discharge in DN neurons. Spike potentials in output neurons of the DN could be often evoked by stimulation of the cerebral cortex, the PN (Figure 3B) and the IO (Figure 4C) and these spikes were generated by EPSPs. ${ }^{13.14}$ Accordingly, the excitatory input from the PN or the IO is not so weak to generate spike activities in DN neurons. In addition, cerebeller nucleus neurons have high spontaneous or background activities. ${ }^{43}$ It has been suggested that the background discharge of cerebellar nucleus neurons is generated by a continuous excitatory barrage along input lines, or nuclear neurons themselves may be generating the discharge without any inputs. ${ }^{4.3}$ Recent study in a slice preparation supports the idea of the latter mechanism by the electroresponsive properties of the membranes of nuclear neurons contributes to the background discharge of nuclear neurons. ${ }^{50}$ Furthermore, Llinás and Mühlethaler ${ }^{51}$ demonstrated the existence of a powerful low threshold $\mathrm{Ca}^{2+}$ dependent spike which triggered a burst of fast $\mathrm{Na}^{+}$-dependent spikes in cerebellar nucleus neurons. Therefore, the firing properties of cerebellar nucleus neurons may be markedly modulated by their resting membrane potential. On account of the underlying spontaneous activity of cerebellar nuclear neurons, changes of spike activities in cerebellar nuclear neurons can be easily generated by slight modulation of the membrane potential by excitatory or inhibitory inputs. Taken together, the excitatory inputs by way of mossy fiber collaterals of the PN and the NRTP origin are thought to be effective enough to modulate 
spike activities of nuclear output neurons. As shown above, inputs from the cerebral cortex are conveyed to the DN by way of several parallel pathways. An important question remains open as to what kinds of information is carried to the DN by way of these individual pathways from the cerebral cortex.

\section{ACKNOWLEDGEMENI}

This research was supported by a grant from the Japanese Ministry of Education, Science and Culture for Scientific Research.

\section{REFERENCES}

1. Cajal S Ramon y. Histologie du System Nerveux de l'Homme etes Vertébrés. Maloine, Paris, 1911.

2. Eccles JC, Ito M, Szentágothai J. The Cerebellum as a Neuronal Machine. Berlin-Heidelberg-New York: Springer, 1967.

3. Ito $M$, Yoshida $M$. The origin of cerebellar-induced inhibition of Deiters neurons. I. Monosynaptic initiation of the inhibitory postsynaptic potentials. Exp Brain Res 1966; 2: 330-349.

4. Ito M, Yoshida M, Obata K, et al. Inhibitory control of intracerebellar nuclei by the Purkinje cell axons. Exp Brain Res 1970; 10: 64-80.

5. Beitz AJ. The topographical organization of the olivo-dentate and dentato-olivary pathways in the cat. Brain Res 1976; 115: 311317.

6. Courville J, Augustine JR, Martel P. Projections from the inferior olive to the cerebellar nuclei in the cat demonstrated by retrograde transport of horseradish peroxidase. Brain Res 1977; 130 405-419.

7. Groenewegen HJ, Voogd J, Freedman SL. The parasagittal zonal organization within the olivocerebellar projection. II. Climbing fiber distribution in the intermediate and hemispheric parts of cat cerebellum. J Comp Neurol 1979; 183: 551-602.

8. Matsushita M, Ikeda M. Olivary projections to the cerebellar nuclei in the cat. Exp Brain Res 1970; 10: 488-500.

9. Eller T, Chan-Palay V. Afferents to the cerebellar lateral nucleus. Evidence from retrograde transport of horseradish peroxidase after pressure injections through micropipettes. J Comp Neurol 1976; 166: 285-302.

10. Oka $\mathrm{H}$, Yoshida $\mathrm{K}$. Yamamoto $\mathrm{T}$, et al. Organization of afferent connections to the lateral and interpositus cerebellar nuclei from the brainstem nuclei: a horseradish peroxidase study in the cat. Neurosci Res 1985: 2: 321-333.

11. Dietrichs E, Bjaale JG, Brodal P. Do pontocerebellar fibers send collaterals to the cerebellar nuclei? Brain Res 1983; 259: 127131.

12. Allen Gl, Gilbert PFC, Marini R, et al. Integration of cerebral and peripheral inputs by interpositus neurons in monkey. Exp Brain Res 1977b; 27: 81-99.

13. Allen GI, Gilbert PFC, Yin TCT. Convergence of cerebral inputs onto dentate neurons in monkey. Exp Brain Res 1978; 32: 151170.

14. Shinoda Y, Sugiuchi Y, Futami T. Excitatory inputs to cerebellar dentate nucleus neurons from the cerebral cortex in the cat. Exp Brain Res 1987; 67: 299-315.

15. Brodal A, Jansen J. The ponto-cerebellar projection in the rabbit and cat. Experimental investigations. J Comp Neurol 1946; 84: $31-118$.

16. Shinoda Y, Sugiuchi Y, Futami T, et al. Axon collaterals of mossy fibers from the pontine nucleus in the cerebellar dentate nucleus. J Neurophysiol 1992; 67: 547-560.

17. Jansen J, Brodal A. Aspects of Cerebellar Anatomy. Oslo: J Chr Grundersen, 1954.

18. Voogd J. The Cerebellum of the Cat. Structure and Fibre Connexions. Proefschr. N.V., Assen: Van Gorcum \& Co. 1964.

19. Eccles JC. The Physiology of Synapses. Berlin: Springer-Verlag, 1964.

20. Hassler WR, Muhs-Clement K. Architektonischer Aufbau des sensori-motorishen und parietalen Cortex der Katze. J Hirnforsch $1964 ; 6: 377-420$
21. Amatuni A, Tarnecki $R$, Wrobel $A$, et al. Interaction on extracerebellar cortical inputs in dentate neurons of the cat. Acta Neurobiol Exp 1981; 41: 373-390.

22. Armstrong DM, Harvey RJ. Responses in the inferior olive to stimulation of the cerebellar and cerebral cortices in the cat. J Physiol (Lond) 1966; 187: 553-574.

23. Van Der Want J, Wiklund L, Guegan M, et al. Anterograde tracing of the rat olivocerebellar system with phaseolus vulgaris leucoagglutinin (PHA-L). Demonstration of climbing fiber collateral innervation of the cerebellar nuclei. J Comp Neurol 1989; 288: $1-18$.

24. Brodal A. Neurological Anatomy in Relation to Clinical Medicine. Oxford: Oxford University Press, 1981 .

25. Brodal P. The corticopontine projection in the cat. I. Demonstration of a somatotopically organized projection from the primary sensorimotor cortex. Exp Brain Res 1968a; 5: 212-237.

26. Brodal P. The corticopontine projection in the cat. II Demonstration of a somatotopically organized projection from the second somatosensory cortex. Arch Ital Biol 1968b; 106 310-332.

27. Mizuno N, Mochizuki K, Akimoto C, et al. Projections from the parietal cortex to the brain stem nuclei in the cat, with special reference to the parietal cerebrocerebellar system. J Comp Neurol 1973; 147: 511-522.

28. Kusama T, Otani K, Kawana E. Projections of the motor, somatic sensory, auditory and visual cortices in cats. In: Tokizane $\mathrm{T}$ Schade' JP, eds. Progress in Brain Research. 21, part A. Amsterdam: Elsevier 1966; 292-322.

29. Brodal A, Brodal P. The organization of the nucleus reticularis tegmenti pontis in the cat in the light of experimental anatomical studies of its cerebral cortical afferents. Exp Brain Res 1971; 13: 90-110.

30. Rüegg DG, Wiesendanger $M$. Corticofugal effects from sensorimotor area $I$ and somatosensory area $I I$ on neurones of the pontine nuclei in the cat. J Physiol (Lond) 1975; 247: 745-757.

31. Allen GI, Korn H, Oshima T, et al. The mode of synaptic linkage in the cerebro-ponto-cerebellar pathway of the cat. II. Responses of single cells in the pontine nuclei. Exp Brain Res 1975; 24: 15 . 36.

32. Allen GI, Oshima T, Toyama $\mathrm{K}$. The mode of synaptic linkage in the cerebro-ponto-cerebellar pathway investigated with intracellular recording from the pontine nuclei cells of the call. Exp Brain Res 1977a; 29: 123-136.

33. Oka H, Sasaki K, Matsuda Y, et al. Responses of pontocerebellar neurones to stimulation of the parietal association and the frontal motor cortices. Brain Res 1975; 93: 399-407.

34. Futami T, Kano M, Sento S, et al. Synaptic organization of the cerebello-thalamo-cerebral pathway in the cat. IIl. Cerebellar input to corticofugal neurons destined for different subcortical nuclei in areas 4 and 6 . Neuroscience Res 1986:3:321-344.

35. Walberg, F. Descending connections to the inferior olive. J Comp Neurol 1956; 104: 77-173.

36. Bishop GA, McCrea RA, Kitai ST. A horseradish peroxidase study of the cortico-olivary projection in the cat. Brain Res 1976: 116: 306-311.

37. Sousa-Pinto A, Brodal A. Demonstration of a somatotopical pattern in the cortico-olivary projection in the cat. An experimentalanatomical study. Exp Brain Res 1969; 8: 364-386.

38. Sousa-Pinto A. Experimental anatomical demonstration of a cortico-olivary projection from area 6 (supplementary motor area?) in the cat. Brain Res 1969; 16:73-83.

39. Saint-Cry JA, Courville J. Projections from the motor cortex, midbrain, and vestibular nuclei to the inferior olive in the cat anatomical organization and funclional correlates. $/ \mathrm{m}$ : Courville J, de Montigny C, Lamarre Y, eds. The Inferior Olivary Nucleus. New York: Raven Press 1980; 97-124.

40. Oka H, Jinnai K, Yamamoto T. The parieto-rubro-olivary pathway in the cat. Exp Brain Res 1979; 37: 115-125.

41. Mano N, Yamamoto K. Simple-spike activity of cerebellar Purkinje cells related to visually guided wrist tracking movement in the monkey. J Neurophysiol 1980; 43: 713-728.

42. Schieber MH, Thach WT. Trained slow tracking. II. Bidirectional discharge patterns of cerebellar nuclear, motor cortex, and spindle afferent neurons. J Neurophysiol 1985; 54: 1228-1270. 
43. Thach WT. Discharge of Purkinje and cerebellar neurons during rapidly alternating arm movement in the monkey. J Neurophysiol 1968; 31: 785-797.

44. Wetts R, Kalaska JF, Smith AM. Cerebellar nuclear cell activity during antagonist cocontraction and reciprocal inhibition of forearm muscles. J Neurophysiol 1985; 54: 231-244.

45. Allen GI, Tsukahara N. Cerebrocerebellar communication systems. Physiol Rev 1974; 54: 957-1006.

46. Chan-Palay V. Cerebellar Dentate Nucleus, Organization, Cytology, and Transmitters. Heidelberg: Springer, 1977.

47. Ito M. The Cerebellum and Neural Control. New York: Raven Press, 1984.

48. Brodal P, Dietrichs E, Walberg F. Do pontocerebellar mossy fibers give off collaterals to the cerebellar nuclei? An experimental study in the cat with implantation of crystalline HRP-WGA. Neurosci Res 1986; 4: 12-24.

49. Gerrits NM, Voogd J. The projection of the nucleus reticularis tegmenti pontis and adjacent regions of the pontine nuclei to the central cerebellar nuclei in the cat. J Comp Neurol 1987; 258: 52-69.

50. Jahnsen H. Electrophysiological characteristics of neurones in the guinea-pig deep cerebellar nuclei in vitro. J Physiol (Lond) 1986; 372: 129-147.

51. Llinás R, Mühlethaler M. Electrophysiology of guinea-pig cerebellar nuclear cells in the vitro brain stem-cerebellar preparation. $J$ Physiol (Lond) 1988; 404: 241-258. 